\title{
Numerical Investigations of Transverse Gradient Undulator Based on Novel Light Sources
}

\author{
Tong Zhang ${ }^{1}$, Guanglei Wang ${ }^{1}$, Haifeng Yao ${ }^{1}$, Dong Wang ${ }^{1}$, Wentao Wang ${ }^{2}$, Cheng Wang ${ }^{2}$, Zhinan Zeng ${ }^{2}$, \\ Jiansheng Liu ${ }^{2}$, Jiuqing Wang ${ }^{3}$ and Shuhong Wang ${ }^{3}$ \\ 1. Shanghai Institute of Applied Physics, Chinese Academy of Sciences, Shanghai 201800, China \\ 2. Shanghai Institute of Optics and Fine Mechanics, Chinese Academy of Sciences, Shanghai 201800, China \\ 3. Institute of High Energy Physics, Chinese Academy of Sciences, Beijing 100049, China
}

Received: May 07, 2014 / Accepted: May 27, 2014 / Published: July 25, 2014.

\begin{abstract}
With the stat-of-the-art laser technique, the quality of electron beam generated from LPA (laser-plasma accelerator) is now becoming much better. The natural merits of electron beam from LPA, e.g., high peak current, ultra-low emittance and ultra-short bunch length, etc., pave the way to the novel light sources, especially in the realm of developing much more compact x-ray light sources, e.g., table-top XFEL (x-ray free-electron laser). However, the radiation power is limited by the rather larger energy spread than conventional radio-frequency electron LINAC (linear accelerator). Luckily, much more power could be extracted by using the undulator with transverse gradient when energy spread effect could be compensated. In this paper, we introduce a novel soft x-ray light source driven by LPA together with TGU (transverse gradient undulator) technique, meanwhile we present a simple idea on how to achieve much higher rep-rate (e.g., $\sim 100 \mathrm{kHz}$ ) FELs (free-electron lasers) boosted by TGU based on storage rings.
\end{abstract}

Key words: FEL, TGU, LPA.

\section{Introduction}

With the advent of the world's first two hard XFELs (x-ray free-electron lasers)-LCLS (Linac Coherent Light Source) [1] and SACLA (Spring-8 Angstrom Compact Free Electron Laser) [2], scientists begins to enjoy much more exciting discoveries. However, the large scale and huge investment of such kind facilities prevent XFEL from being popular worldwide, especially in the much smaller university laboratories. One of the most important aspects is the rather longer RF (radio-frequency) LINAC (linear accelerator), since tens of $\mathrm{GeV}$ electron beam is required in the XFEL with the acceleration gradient of tens of $\mathrm{MeV}$ per meter.

On the other hand, the laser plasma acceleration technique could generate electron beam with energy

Corresponding author: Dong Wang, research professor, Ph.D., research fields: free-electron laser, particle accelerator. E-mail: wangd@sinap.ac.cn. of $\mathrm{GeV}$ in just centimeter scale [3], which absolutely enlightens the FEL community to build much more compact XFELs by simply replacing the large LINAC with LPA (laser plasma accelerator). Nevertheless, the electron beam quality from LPA could not be controlled as much ideally as the conventional RF LINAC, it is reported that the LPA could provide electron beam with the energy of $\sim \mathrm{GeV}$ [3], normalized transverse emittance of $\sim 0.1 \mu \mathrm{m}$ [4], peak current of several kilo Amperes, bunch length of tens of femto-seconds or even shorter, but relative energy spread of a few percent level which could limit the maximum FEL output power [5].

Recently, Huang et al. [6] proposed an idea to compensate the energy spread effect in the LPA driven high-gain FELs, which reported that by properly transversely dispersing the LPA electron beam, the percent level energy spread in the longitudinal phase space (i.e., $\gamma-t$ ) could be 
transformed into the transversal displacement, that means by using undulators with proper transverse gradient the FEL resonant phenomenon could be maintained for electrons with different energies. The essence of TGU (transverse gradient undulator) application in the high-gain FEL relies on the fact that sacrificing the transverse current density of electron beam to increase the final extracted FEL power.

Since TGU could be used as the energy spread compensator, in the diffraction-limited storage rings, straight TGU radiator by-pass line could be used to generate FELs with high rep-rate [7, 8]. Moreover, we can take the advantage of the rather larger acceptance of TGU to increase the rep-rate by slowly damping.

In this paper, we briefly review the TGU principle on energy spread suppression, then three novel light sources based on TGU is presented and the numerical simulations is mainly focused. The paper is organized as follows: Section 2 discusses a soft $\mathrm{x}$-ray FEL driven by TGU and LPA; Section 3 presents the basic idea of a high rep-rate storage ring based FEL powered by TGU; and Section 4 gives conclusions.

\section{Soft X-ray FEL Driven by LPA and TGU}

The theory of TGU could be simply linked by Eqs. (1) and (2):

$$
\begin{aligned}
& a_{u}(x)=a_{u}(1+a x) \\
& x=\eta \frac{\Delta \gamma}{\gamma}
\end{aligned}
$$

where, $a_{u}$ is the normalized undulator parameter, $x$ is the transverse deviation, $\alpha$ is the transverse field gradient, $\eta$ is the transverse dispersion, $\Delta \gamma / \gamma$ is the energy deviation. By introducing the FEL resonant equation: $\lambda_{s}=\lambda_{u} /\left(2 \gamma^{2}\right)\left(1+a_{u}{ }^{2}\right)$, the relationship between $\Delta \gamma / \gamma$ and $\Delta a_{u} / a_{u}$ is found as: $\Delta \gamma / \gamma=a_{u}^{2} /\left(1+a_{u}^{2}\right) \cdot \Delta a_{u} / a_{u}$.

Then the compromise condition between the field gradient and transverse dispersion is worked out as:

$$
\alpha \cdot \eta=\frac{1+a_{u}{ }^{2}}{a_{u}{ }^{2}}
$$

Here we would like to present the numerical simulations of an LPA driven XFEL which equipped with TGU at SIOM (Shanghai Institute of Optics and Fine Mechanics), Chinese Academy of Sciences. The laser plasma accelerator is now under operation at SIOM, electron beam with nearly $1 \mathrm{GeV}$ and energy spread about 3\%, peak current of several $\mathrm{kA}$ is generated [9]. The next plan is to construct a soft $\mathrm{x}$-ray light source driven by LPA with the name of SIOM-FEL, which is also a collaborated project with SINAP (Shanghai Institute of Applied Physics).

It is well known that the longitudinal coherency of FEL could be improved by seeding the electron beam with external conventional optical laser before entering into the radiator, e.g., the famous schemes HGHG (high-gain harmonic generation) [10] and EEHG (echo-enabled harmonic generation) [11], and the newly proposed cooled HGHG [12], etc. The next phase of SIOM-FEL will introduce HHG (high-order harmonic generation) seed laser which is already in the same building of laser plasma accelerator. The HHG seed reported can generate coherent light source around 30 from $800 \mathrm{~nm}$ optical laser. In phase-II of SIOM-FEL, TGU is also taken into account, newly designed and built TGU by SINAP will be used as the radiator to generate much more intense FEL pulses.

Here, numerical simulations are focused on SIOM-FEL seeded by HHG seed and boosted by TGU. Fig. 1 shows the schematic layout of SIOM-FEL. The main parameters of SIOM-FEL could be found in Table 1.

The numerical simulations are studied by the well benchmarked FEL code - GENESIS [13], which was modified for including the undulator field transverse gradient. The compromise between the transverse

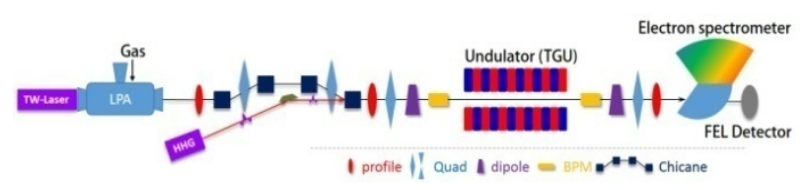

Fig. 1 Schematic layout of SIOM-FEL. The layout shows five key sections, the laser plasma accelerator driven by intense Terawatt laser, the electron beam transportation line, the HHG seeding line, radiators and diagnostics for electron beam \& photon. 
Table 1 Main parameters of SIOM-FEL.

\begin{tabular}{llll}
\hline Parameter & Symbol & Value & Unit \\
\hline Beam energy & $E_{b}$ & 400 & $\mathrm{MeV}$ \\
Energy spread & $\sigma_{\gamma}$ & 4 & $\mathrm{MeV}$ \\
Emittance & $\varepsilon_{n}$ & 0.1 & $\mathrm{~mm} \cdot \mathrm{mrad}$ \\
Beam size & $\sigma_{x}$ & 10 & $\mu \mathrm{m}$ \\
Peak current & $I_{p k}$ & $1-2$ & $\mathrm{kA}$ \\
E-charge & $Q$ & $\sim 10-20$ & $\mathrm{pC}$ \\
Bunch length & $\sigma_{\tau}$ & 10 & $\mathrm{fs}$ \\
Seed wavelength & $\lambda_{\mathrm{HHG}}$ & 30 & $\mathrm{~nm}$ \\
Seed Rayleigh range & $Z_{R}$ & 1 & $\mathrm{~m}$ \\
Seed peak power & $P_{\text {seed }}$ & 10 & $\mathrm{MW}$ \\
Radiator period length & $\lambda_{r}$ & 20 & $\mathrm{~mm}$ \\
FEL wavelength & $\lambda_{s}$ & 30 & $\mathrm{~nm}$ \\
\hline
\end{tabular}

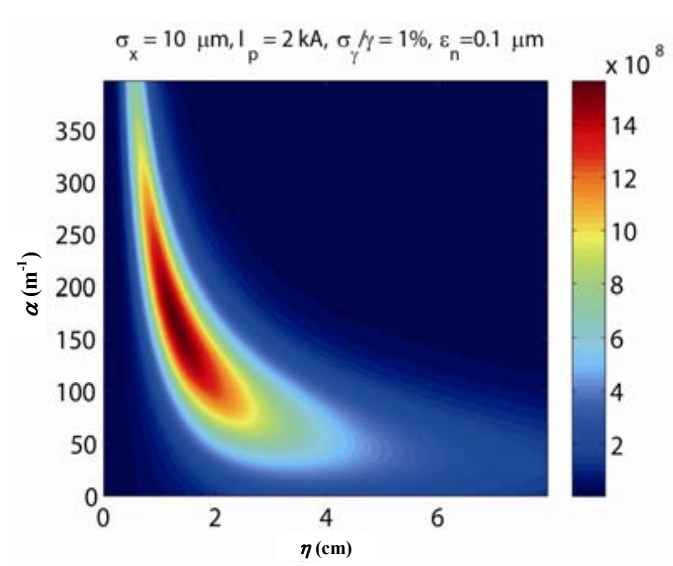

(a)

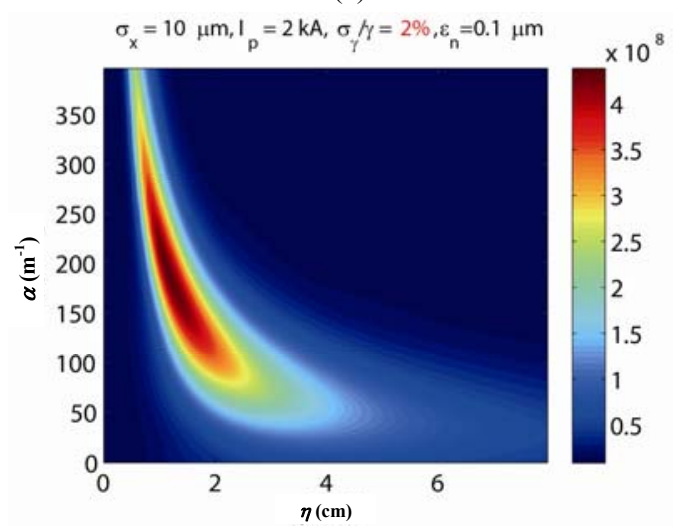

(b)

Fig. 2 Two-dimensional optimization of $\alpha$ and $\eta$ with the electron parameters of $\sigma_{x}=10 \mu \mathrm{m}, I_{p k}=2 \mathrm{kA}$ and $\varepsilon_{n}=0.1$ $\mu \mathrm{m}$, the relative energy spread used here is (a) $1 \%$ and (b) $2 \%$, respectively.

dispersion $\eta$ and field gradient $\alpha$ should be found by two-dimensional optimization, as Fig. 2 shows, it is clearly that the optimal FEL output power decreases from $1.6 \mathrm{GW}$ to only $0.4 \mathrm{GW}$ for the case of $1 \%$ and
$2 \%$ relative energy spread, respectively.

To envision the promising future, here we fix the energy spread to be $1 \%$, and apply the optimal $(\alpha, \eta)=$ $\left(164 \mathrm{~m}^{-1}, 1.32 \mathrm{~cm}\right)$, which means the poles' tilt angle of $21^{\circ}$ for Halbach permanent undulator [14] is required, however the numerical simulation shows that with the much more practical tilt angle of $7^{\circ}(\alpha=$ $50 \mathrm{~m}^{-1}$ ) the radiation power still could be up be 200 MW.

The time-dependent FEL simulations are also studied for the cases with TGU and normal undulator, respectively; both are seeded by HHG source. Fig. 3 shows that after 150 periods radiator, the HHG seed is amplified over 140 times by TGU, while almost only 2 times by normal undulator, evident exponential growth could be seen for TGU case.

It is also noted that even if the electron beam from LPA with worse quality, e.g., $\sigma_{x}=50 \mu \mathrm{m}, \varepsilon_{n}=1.0 \mu \mathrm{m}$, TGU still could amplified the seed by about 10 times.

\section{High Rep-Rate XFEL Driven by USR and TGU}

The BAPS (Beijing Advanced Photon Source) is a major facility being planned for a new research center in northeast Beijing by Chinese Academy of Sciences. The schematic layout of BAPS could be found in Fig. 4 , one can see two large rings with the circumference of about $1.2 \mathrm{~km}$, the different vertical latitude allows for the light beam extraction from both inner and outer rings [15]. Here we consider the FEL option for BAPS, which emittance could be lower down to diffraction-limited, by using TGU in the bypass straight line, the energy spread effect could be compensated thus to achieve much higher FEL power output.

Below shows the main parameters when running under the high rep-rate FEL mode, in which TGU is used as the main straight radiator lines, with the aim to get $\geq 100 \mathrm{kHz}$ rep-rate by slowly damping.

We introduce the coupling factor of $1 \%$ into the electron beam, and the normalized emittance used 
here is $\varepsilon_{x}=0.6 \mu \mathrm{m}, \varepsilon_{y}=0.006 \mu \mathrm{m}$. On entering the straight bypass line, the electron beam is first vertically dispersed to form round shape transverse beam size, then the FEL amplified along TGU. The optimization of transverse gradient and transverse dispersion could be found in Fig. 5. The FEL power gain curve could be found under different peak currents in Fig. 6, also shows the cases with normal undulators.

One of the greatest advantages of storage ring based FELs is the high repetition rate, which mean higher average brilliance. However, the FEL process in the bypass straight line will absolutely destroy the electron beam quality equilibrium, extra time is required to restore such equilibrium state; the damping time is usually of millisecond order, which means the rep-rate should drop from $\mathrm{MHz}$ to $\mathrm{kHz}$ level. Here we
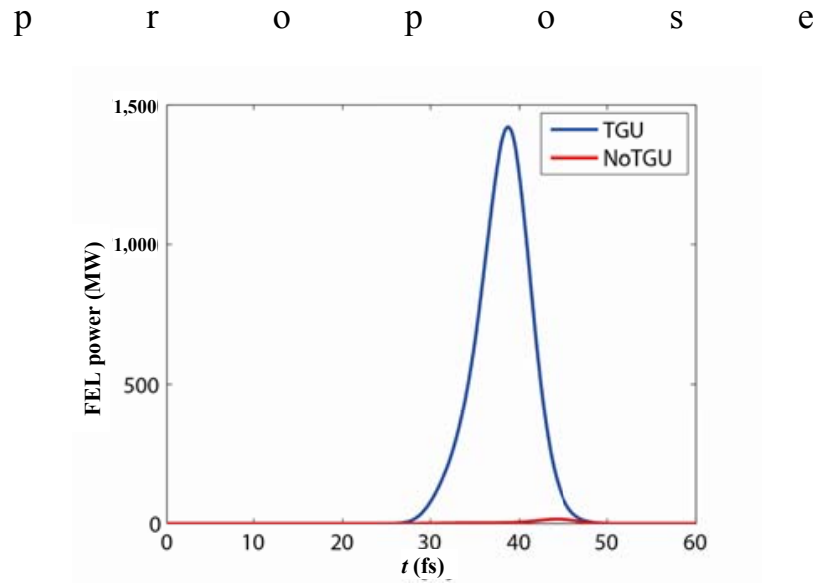

(a)

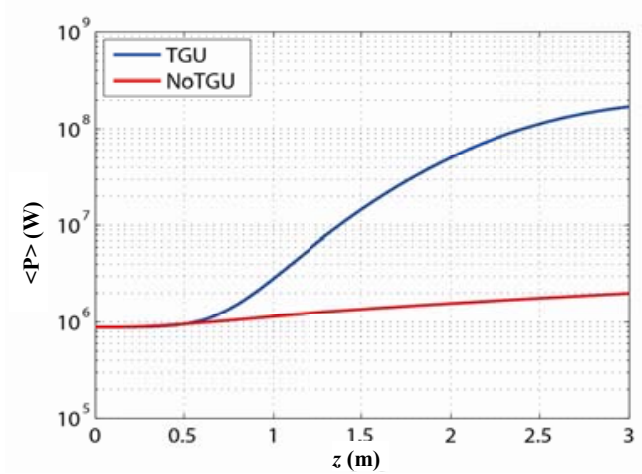

(b)

Fig. 3 (a) Simulated FEL pulse profile and (b) gain curve with the case of TGU and normal undulator, electron parameters used here are $\sigma_{x}=10 \mu \mathrm{m}, I_{p k}=2 \mathrm{kA}, \varepsilon_{n}=0.1$ $\mu \mathrm{m}$ and $\sigma_{\gamma} / \gamma=1 \%$.

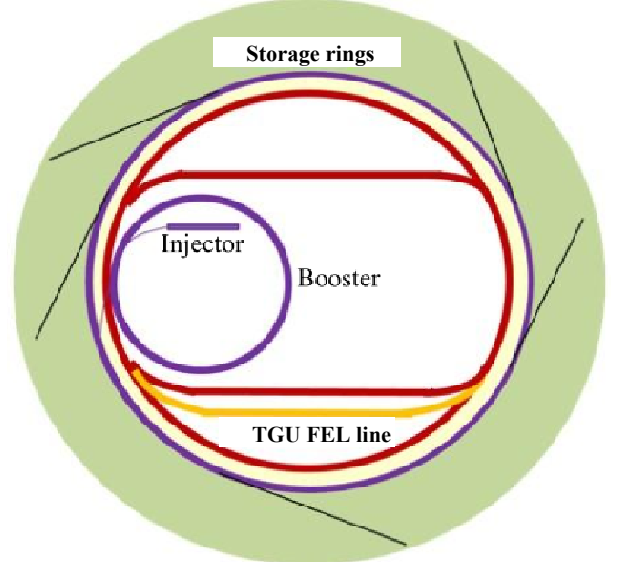

Fig. 4 Schematic layout of BAPS, with the beam energy of $5 \mathrm{GeV}$, the yellow bypass straight line (total length could be up to 100-200 $\mathrm{m}$ ) is designed for generating FELs.

Table 2 Parameters of BAPS-FEL.

\begin{tabular}{llll}
\hline Parameter & Symbol & Value & Unit \\
\hline Beam energy & $E_{b}$ & 3.0 & $\mathrm{GeV}$ \\
Energy spread & $\sigma_{\gamma} / \gamma$ & 0.1 & - \\
Peak current & $I_{p k}$ & $100-300$ & $\mathrm{~A}$ \\
Undulator period & $\lambda_{u}$ & 0.03 & $\mathrm{~m}$ \\
Undulator parameter & $K_{0}$ & 1.61 & - \\
Undulator length & $L_{u}$ & 180 & $\mathrm{~m}$ \\
Average beta x & $<\beta_{x}>$ & 70 & $\mathrm{~m}$ \\
Average beta y & $<\beta_{y}>$ & 20 & $\mathrm{~m}$ \\
Transverse dispersion & $\eta$ & 4.5 & $\mathrm{~cm}$ \\
Transverse gradient & $\alpha$ & 40 & $\mathrm{~m}^{-1}$ \\
FEL wavelength & $\lambda_{\mathrm{FEL}}$ & 1.0 & $\mathrm{~nm}$ \\
FEL peak power & $P_{\mathrm{FEL}}$ & $\sim 200$ & $\mathrm{MW}$ \\
FEL pulse energy & $W_{\mathrm{FEL}}$ & $\sim 200$ & $\mu \mathrm{J}$ \\
FEL flux & $F_{\mathrm{FEL}}$ & $1 \times 10^{12}$ & $\# / \mathrm{pulse}$ \\
\hline
\end{tabular}

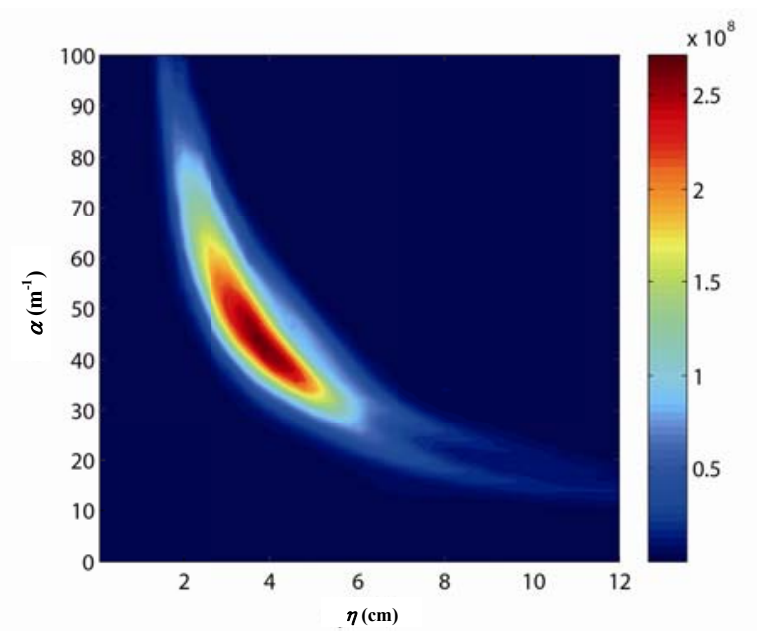

Fig. 5 Optimization between the transverse gradient and dispersion for BAPS-FEL. 


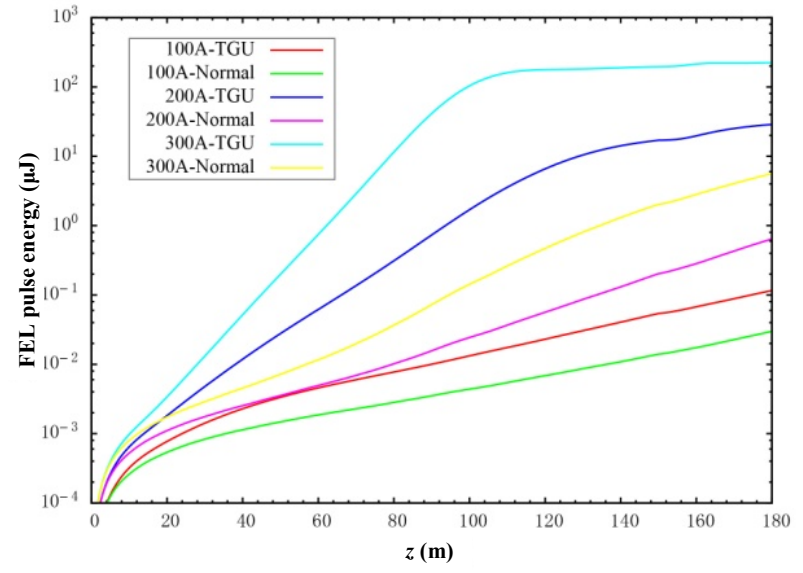

Fig. 6 Time-dependent simulated FEL power gain curve with different peak currents.
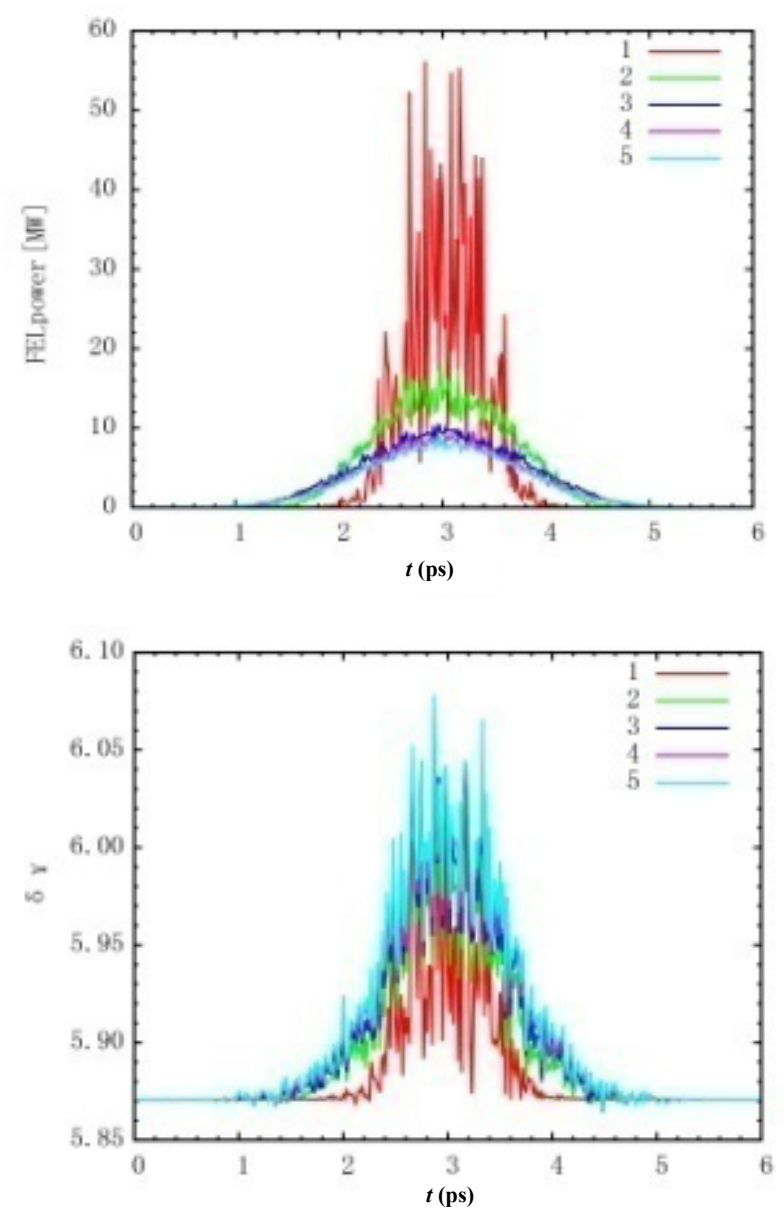

Fig. 7 Time-dependent simulated FEL power and energy spread within 5 trips along TGU by pass line (no damping).

that the rep-rate could be increased by letting the electron beam passes through the TGU line several times before damping, or slowly damping, since TGU is an energy spread compensator, the FEL power should almost be of the same order level within the round trip passing TGU (Fig. 7). The consequence is that the rep-rate could be increased by 1-2 orders, i.e., the goal of BAPS-FEL to $10-100 \mathrm{kHz}$ rep-rate. Table 2 shows the main parameters of such kind of light source.

\section{Conclusions}

In this paper, the application of transverse gradient undulator in the free-electron laser has been carefully investigated by numerical simulation approach. The much more compact $\mathrm{x}$-ray free-electron laser-SIOM-FEL could has the ability to generate several hundred MW FEL power if TGU is properly used. The high repetition rate storage ring based FEL could also been working in the high-gain regime, even higher rep-rate could be achieved if let the electron beam slowly damp in the bypass TGU line and storage rings. The preliminary numerical simulations of BAPS-FEL indicate that the rep-rate of BAPS-FEL could be up to no less than $50 \mathrm{kHz}, 100 \mathrm{kHz}$ is still the goal to achieve. Finally, it should be optimistic that beam quality of LPA especially the energy spread once drops down to $0.1 \%$ region, the table-top XFEL driven by LPA will open up new realms.

\section{Acknowledgments}

The authors would like to thank Huang, Z., and Ding, Y. from SLAC and Feng, C., Deng, H., Lan, T., Shen, L., Wang, X. and Liu, B. from SINAP for helpful discussions. The authors are grateful for the support of Major State Basic Research Development Program of China (No. 2011CB808300), and Natural Science Foundation of China (No. 11075199).

\section{References}

[1] Emma, P. 2010. "First Lasing and Operation of an Ångstrom-Wavelength Free-Electron Laser." Nature Photonics 4: 641-47.

[2] Ishikawa, T. 2012. "A Compact X-Ray Free-Electron Laser Emitting in the Sub-ångström Region.” Nature 
Photonics 6: 540-44.

[3] Leemans, W. P. 2006. "GeV Electron Beams from a Centimetre-Scale Accelerator." Nature Physics 2: 696-99.

[4] Fritzler, S. 2004. "Emittance Measurements of a Laser-Wakefield-Accelerated Electron Beam." Phys. Rev. Lett. 92: 165006.

[5] Lundh, O. 2011. "Few Femtosecond, Few Kiloampere Electron Bunch Produced by a Laser-Plasma Accelerator." Nature Physics 7: 219-22.

[6] Huang, Z. 2012. "Compact X-Ray Free-Electron Laser from a Laser-Plasma Accelerator Using a Transverse-Gradient Undulator." Phys. Rev. Lett. 109: 204801.

[7] Cai, Y. 2013. "An X-Ray Free Electron Laser Driven by an Ultimate Storage Ring." SLAC-PUB-15380.

[8] Ding, Y. 2013. "High-gain X-Ray FELs Using a Transverse Gradient Undulator in an Ultimate Storage Ring." In Proceedings of IPAC 2013, 2286-88.

[9] Liu, J. S. 2011. “All-Optical Cascaded Laser Wakefield
Accelerator Using Ionization-Induced Injection.” Phys. Rev. Lett. 107: 035001.

[10] Yu, L. H. 2000. "High-Gain Harmonic-Generation Free-Electron Laser." Science 289: 932-34.

[11] Stupakov, G. 2009. "Using the Beam-Echo Effect for Generation of Short-Wavelength Radiation." Phys. Rev. Lett. 102: 074801.

[12] Deng, H. X., and Feng, C. 2013. "Using Off-Resonance Laser Modulation for Beam-Energy-Spread Cooling in Generation of Short-Wavelength Radiation.” Phys. Rev. Lett. 111: 084801.

[13] Reiche, S. 1999. "GENESIS 1.3: A Fully 3D Time-Dependent FEL Simulation Code." Nucl. Instr. Meth. A. 429: 243-48.

[14] Halbach, K. 1983. "Permanent Magnet Undulators." $J$. Phys. Colloques 44 (C1) 211-16.

[15] Wang, D. 2013. "An Overview of Light Source Development in Asia." In Proceedings of IPAC2013, 4005-09. 